\title{
Forma analítica de la extensión de funcionales lineales en espacios normados
}

\author{
Analytical form of extension of linear functionals in normed spaces
}

\author{
${ }^{1}$ Jhony Alfonso Chávez Delgado, ${ }^{2}$ Joel Mike Flores Calisaya
}

\begin{abstract}
RESUMEN
El objetivo de este artículo de investigación es establecer condiciones para extender una funcional lineal de un subespacio vectorial a todo el espacio normado preservando las propiedades básicas de dicho conjunto de donde se extendió y que en la actualidad tiene aplicaciones en otras áreas de la matemática, por ejemplo: análisis complejo, teoría de juegos y en el análisis convexo. Se determinó que es posible extender una funcional lineal preservando la linealidad, la relación de orden y el valor de la funcional, para todo elemento de un subespacio vectorial E sobre el campo de los números reales o complejos. Se empleó los métodos deductivo e inductivo para extender una funcional lineal de un espacio vectorial a todo el espacio normado y para contrastar el funcionamiento de estas condiciones, se analizó los teoremas de extensión de funcionales lineales en los espacios normados para el caso real y para el caso complejo las cuales se derivan en proposiciones.
\end{abstract}

Palabras Clave: espacios normados, teorema de extensión de funcionales lineales, espacios separables.

\begin{abstract}
The objective of this research article is to establish conditions to extend a linear functional of a vector subspace to the entire normed space preserving the basic properties of said set from where it was extended and that currently has applications in other areas of mathematics for example : complex analysis, game theory and convex analysis. It was determined that it is possible to extend a linear functional preserving the linearity, the order relationship and the value of the functional, for all element of a vector subspace $\mathrm{G}$ of a vector space over the field of the real or complex numbers. Were used the deductive and inductive methods to extend a linear functional of a vector space to the entire normed space and to contrast the operation of these conditions, the linear functional extension theorems in the normed spaces were analyzed for the real case and for the complex case which are derived in propositions.
\end{abstract}

Keywords: normed spaces, linear functional extension theorem, separable spaces.

\footnotetext{
${ }^{1}$ Universidad Nacional Jorge Basadre Grohmann. Tacna-Perú. E-mail: jchavez@unjbg.edu.pe

${ }^{2}$ Universidad Nacional Jorge Basadre Grohmann. Tacna-Perú. E-mail: 4030jf@gmail.com 


\section{INTRODUCCIÓN}

En el análisis funcional, la extensión de funcionales lineales es una herramienta importante tanto en su teoría axiomática como en sus corolarios y sus aplicaciones. Permite extender cualquier funcional lineal continúo definido en un subespacio normado al espacio normado que lo contiene. Debe su nombre a Hahn y Stefan Banach quienes probaron este teorema independientemente en la década de 1920. El teorema aparece en matemáticas en formas diversas, tanto analíticas como geométricas.

La esencia del teorema de extensión de funcionales, en su versión para espacios normados, es que la funcional lineal continúa definido en un subespacio vectorial $G$ de un espacio normado $E$ puede ser extendido a todo el espacio $E$ preservando la linealidad, continuidad y así mismo el valor de la norma. La idea fundamental de la demostración del teorema de la extensión de funcionales lineales es un argumento analítico que muestra que tal extensión es posible de $G$ para $G \oplus[v]$ con $v \in G$, (Botelho, 2012).

En este artículo de investigación se demostró la forma analítica de los teoremas de extensión para el caso real y complejo, y se aplicó el teorema de extensión de funcionales para espacios separables. Este resultado dice, esencialmente, que si $G$ es un subespacio de un espacio normado $E$ sobre un campo $\mathbb{K}$ y sea $\varphi: G \mapsto \mathbb{K}$ una funcional lineal continua. Entonces existe una funcional lineal continua $\tilde{\varphi}: E \mapsto \mathbb{K}$ cuya restricción a $G$ coincide $\operatorname{con} \varphi$ y $\|\tilde{\varphi}\|=\|\varphi\|$.

Este articulo contribuye a investigar la forma analítica de la extensión de funcionales lineales en espacios normados que ha sido estudiada por investigadores como Llorens (2016); Laratonda (2017); Cabello (2009); Baggett (1992); Wawrzynczyk (1993) y otros.

y continua, es decir $\forall x_{0} \in V, \epsilon>0 \exists \delta>0$ tal que

$$
\left\|T(x)-T\left(x_{0}\right)\right\|<\epsilon,
$$

siempre que $\left\|x-x_{0}\right\|<\delta, \forall x \in V$.

(Gatica, 2012).

\section{Caracterización del operador lineal continúo}

Definición (Función Lipschitz). Una función $f: M \mapsto N$ entre espacios métricos es Lipschitziana si existe una constante $L \geq 0$ tal que

\section{ESPACIO DE BANACH}

Definición (Norma). Sea $X$ un espacio vectorial sobre $\mathbb{K}(\mathbb{R} o \mathbb{C})$. Una norma sobre $X$ es una aplicación

$$
\|.\|: X \mapsto \mathbb{R}_{+}=[0,+\infty[
$$

, tal que

(1) $\|x+y\| \leq\|x\|+\|y\|, \forall x, y \in X$.

(2) $\|\alpha x\|=|\alpha|\|x\|, \forall x \in X, \forall \alpha \in \mathbb{K}$.

(3) $\|x\|=0 \Rightarrow x=0, \forall x \in X$.

(Kreyzig, 1989).

Definición (Espacio normado). Si $X$ es un espacio vectorial y $\|\|:. X \mapsto \mathbb{R}_{+}$una norma sobre $X$. Se dice que $X \equiv(X,\|\|$.$) es un espacio normado.$

(Kreyzig, 1989).

Definición (Espacio de Banach). Si $X$ es un espacio normado y $d(x, y)=\|x-y\|$, entonces $X$ es un espacio de Banach si $(X, d)$ es completo.

(Kreyzig, 1989).

\section{Operador lineal continúo}

Definición (Operador lineal continuo). Un operador lineal continuo de un espacio normado $V$ en un espacio normado $W$, ambos sobre el mismo cuerpo $\mathbb{K}$, es una función $T: V \mapsto W$ que es lineal, es decir:

a) $T(x+y)=T(x)+T(y), \forall x, y \in V$,

b) $T(\alpha x)=\alpha T(x), \forall \alpha \in \mathbb{K}, x \in V$.

$$
d(f(x), f(y)) \leq L d(x, y), \forall x, y \in M
$$

(Cabello, 2009).

Definición (Función uniformemente continua). Una función $f: M \mapsto N$ entre espacios métricos es uniformemente continua si

$\forall \epsilon>0 \exists \delta>0$ tal que

$$
\begin{gathered}
\forall(x, y) \in M, d(x, y)<\delta \\
\Rightarrow d(f(x), f(y))<\epsilon .
\end{gathered}
$$

(Kreyzig, 1989).

Lema (Lema de Zorn). Todo conjunto ordenado, inductivo y no vacío admite un elemento máximo.

(Brézis ,1964) 


\section{MATERIALES Y MÉTODOS}

Este artículo corresponde a las ciencias formales y como material de estudio consideramos funcionales lineales continúas definidas en un espacio vectorial con valores reales o complejos.

Se empleó para el desarrollo de este artículo el método lógico deductivo apoyado en la teoría axiomática para demostrar la forma analítica de la extensión de funcionales en los espacios normados, comenzando con las afirmaciones de la forma analítica de la extensión de funcionales, en el caso real y culminando con el caso complejo, pasando por las aplicaciones del teorema de la extensión de funcionales para espacios normados.

\section{RESULTADOS}

\section{Extensión de funcionales Caso real}

funcional lineal $\tilde{\varphi}: E \mapsto \mathbb{K}$ que extiende $\varphi$ a $E$ y que satisface

$$
|\tilde{\varphi}(x)| \leq p(x), \forall x \in E .
$$

Extensión de las funcionales lineales en espacios normados

Sea $E$ un espacio normado. Para todo $x_{0} \in E, x_{0} \neq 0$, entonces existe una funcional lineal $\varphi \in E^{\prime}$ talque $\|\varphi\|=1$ у $\varphi\left(x_{0}\right)=\left\|x_{0}\right\|$.

Botelho (2012)

\section{DISCUSIóN}

\section{Extensión de funcionales-Caso real}

Proposición (Extensión de funcional real). Sean $E$ un espacio vectorial sobre el cuerpo de los reales y $p: E \mapsto$ $\mathbb{R}$ una función que satisface

$$
\begin{aligned}
& p(a x)=a p(x), \forall a>0, \forall x \in E \mathrm{y} \\
& p(x+y) \leq p(x)+p(y), \forall x, y \in E .
\end{aligned}
$$

Sean $E$ un espacio vectorial sobre el cuerpo de los reales y $p: E \mapsto \mathbb{R}$ una función que satisface

$$
\begin{aligned}
& p(a x)=a p(x), \forall a>0, \forall x \in E \mathrm{y} \\
& p(x+y) \leq p(x)+p(y), \forall x, y \in E .
\end{aligned}
$$

Sean $G$ un subespacio vectorial de $E$ y $p: G \mapsto \mathbb{R}$ una funcional lineal tal que

$\varphi(x) \leq p(x), \forall x \in G$. Entonces existe una funcional lineal $\tilde{\varphi}: E \mapsto \mathbb{R}$ que extiende $\varphi$, es decir $\tilde{\varphi}=\varphi \forall x \in$ $G$ y que satisface $\tilde{\varphi} \leq p(x) \forall x \in E$.

Botelho (2012)

\section{Extensión de funcionales Caso real y complejo}

Sea $E$ un espacio vectorial sobre el cuerpo $\mathbb{K}=\mathbb{R}$ o $\mathbb{C}$ y $p: E \mapsto \mathbb{R}$ una función que satisface

$$
\begin{aligned}
& p(a x)=|a| p(x) \forall a \in \mathbb{K}, \forall x \in E \\
& p(x+y) \leq p(x)+p(y) \quad \forall x, y \in E
\end{aligned}
$$

Si $G \subset E$ es un espacio vectorial y $\varphi: G \mapsto \mathbb{K}$ es una funcional lineal tal que $|\varphi(x)| \leq p(x)$ $\forall x \in G$, entonces existe una

Sean $G$ un subespacio vectorial de $E$ y $p: G \mapsto \mathbb{R}$ una funcional lineal tal que $\varphi(x) \leq p(x), \forall x \in G$. Entonces existe una funcional lineal $\tilde{\varphi}: E \mapsto \mathbb{R}$ que extiende $\varphi$, es decir $\tilde{\varphi}=\varphi \forall x \in G$ y que satisface $\tilde{\varphi} \leq$ $p(x) \forall x \in E$.

\section{Prueba}

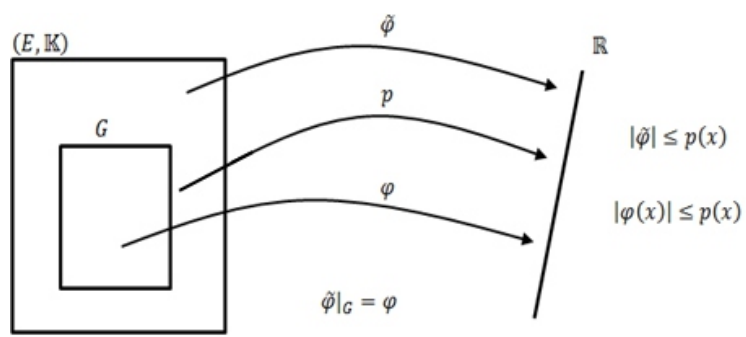

Figura1. Teorema de la extensión

Fuente: Elaboración propia

Considere la familia $P$ de funcionales lineales definidas en un subespacio de $E$ que contienen $G$ :

Sea $P$ el conjunto

$$
\left\{\begin{array}{c}
\phi: D(\phi) \subseteq E \mapsto \mathbb{R} \text { es subespacio vectorial de } E, \\
\phi \text { es lineal, } G \subseteq D(\phi), \phi(x)=\varphi(x) \forall x \in G \\
y \phi(x) \leq p(x) \forall x \in D(\phi)
\end{array}\right\}
$$

En $P$, definimos la relación de orden parcial

$\phi_{1} \leq \phi_{2} \Leftrightarrow D\left(\phi_{1}\right) \subseteq D\left(\phi_{2}\right), \mathrm{y}$

$\phi_{2}$ extiende $\phi_{1}$, es decir $\phi_{2}(x)=\phi_{1}(x) \forall x \in D\left(\phi_{1}\right)$.

Note que $P$ es no vacío porque $\varphi \in P$. Veamos que todo subconjunto totalmente ordenado de $P$ admite una cota superior. En efecto, dado $Q \subseteq P$ totalmente ordenado, defina

$\phi: D(\phi) \mapsto \mathbb{R}$, por $D(\phi)=\bigcup_{\mathrm{O} \in Q} D(0)$ y $\phi(x)=$ $\mathrm{O}(x)$ si $x \in D(0)$.

Note que una buena definición de $\phi$ recorre la ordenada total de $Q$. Se sigue que $\phi \in P$ y que $\phi$ es una cota superior para $Q$. Podemos usar el Lema de Zorn y concluir que $P$ admite un elemento maximal, que será 


$$
\begin{aligned}
& \sup _{x \in D(\widetilde{\varphi})}\left\{\tilde{\varphi}(x)-p\left(x-x_{0}\right)\right\} \\
\leq & \inf _{x \in D(\widetilde{\varphi})}\left\{p\left(x-x_{0}\right)-\tilde{\varphi}(x)\right\}
\end{aligned}
$$

Luego $\forall x, y \in D(\tilde{\varphi})$ tenemos

$$
\begin{aligned}
\tilde{\varphi}(x)+\tilde{\varphi}(y)=\tilde{\varphi} & (x+y) \leq p(x+y) \\
& =p\left(x+y+x_{0}-x_{0}\right) \\
& \leq p\left(x+x_{0}\right)+p\left(y-x_{0}\right),
\end{aligned}
$$

y consecuentemente

$$
\begin{array}{r}
\tilde{\varphi}(x)-p\left(y-x_{0}\right) \\
\leq p\left(x+x_{0}\right)-\tilde{\varphi}(y) \forall x, y \in D(\tilde{\varphi}) .
\end{array}
$$

Por tanto

- Para $t>0$,

$$
\begin{array}{r}
\tilde{\phi}\left(x+t x_{0}\right)=\tilde{\phi}\left(t\left(\frac{x}{t}+x_{0}\right)\right)=t \tilde{\phi}\left(\frac{x}{t}+x_{0}\right) \\
\leq t p\left(\frac{x}{t}+x_{0}\right)=p\left(x+t x_{0}\right) .
\end{array}
$$

- Para $t<0$,

$$
\begin{gathered}
\tilde{\phi}\left(x+t x_{0}\right)=\tilde{\phi}\left(-t\left(\frac{x}{-t}-x_{0}\right)\right)=-t \tilde{\phi}\left(\frac{x}{-t}-x_{0}\right) \\
\leq-t p\left(\frac{-x}{t}-x_{0}\right)=p\left(x+t x_{0}\right)
\end{gathered}
$$

- Para $t=0$,

denotado por $\tilde{\varphi}$. Observe que para obtener el resultado basta mostrar que $D(\tilde{\varphi})=E$. Para eso supongamos que $D(\tilde{\varphi}) \neq E$.

En este caso podemos escoger $x_{0} \in E-D(\tilde{\varphi})$ y definir $\tilde{\phi}: D(\tilde{\phi}) \mapsto \mathbb{R}$ por

$$
\begin{gathered}
D(\tilde{\phi})=D(\tilde{\varphi})+\left[x_{0}\right], \\
\tilde{\phi}\left(x+t x_{0}\right)=\tilde{\varphi}(x)+t \alpha,
\end{gathered}
$$

donde $\alpha$ es una constante que será escogido posteriormente de forma a garantizar $\tilde{\phi} \in P$.

Queremos que $\alpha$ satisfaga las siguientes desigualdades:

$$
\begin{aligned}
\tilde{\varphi}(x)+\alpha= & \tilde{\phi}\left(x+x_{0}\right) \leq p\left(x+x_{0}\right) ; \\
& \forall x \in D(\tilde{\varphi}) . \\
\tilde{\varphi}(x)-\alpha= & \tilde{\phi}\left(x-x_{0}\right) \leq p\left(x-x_{0}\right) ; \\
& \forall x \in D(\tilde{\varphi}) .
\end{aligned}
$$

Escogemos $\alpha$ de modo que

$$
\tilde{\phi}\left(x+t x_{0}\right)=\tilde{\phi}(x)=\tilde{\varphi}(x) \leq p(x)=p\left(x+t x_{0}\right) .
$$

Tenemos que $\phi \in P, \tilde{\varphi} \leq \tilde{\phi}$ y $\tilde{\varphi} \neq \phi$. $P$, admite un maximal de $\tilde{\varphi}$, luego $D(\tilde{\varphi})=E$.

\section{Extensión de funcionales Caso real y complejo}

Proposición (Extensión de funcionales real y complejo). Sea $E$ un espacio vectorial sobre el cuerpo $\mathbb{K}=\mathbb{R}$ o $\mathbb{C}$ y $p: E \mapsto \mathbb{R}$ una función que satisface

$$
\begin{aligned}
& p(a x)=|a| p(x) \forall a \in \mathbb{K}, \forall x \in E \\
& p(x+y) \leq p(x)+p(y) . \forall x, y \in E
\end{aligned}
$$

Si $G \subset E$ es un espacio vectorial y

$\varphi: G \mapsto \mathbb{K}$ es una funcional lineal tal que $|\varphi(x)| \leq p(x)$ $\forall x \in G$, entonces existe una funcional lineal $\tilde{\varphi}: E \mapsto \mathbb{K}$ que extiende $\varphi$ a $E$ y que satisface

$$
|\tilde{\varphi}(x)| \leq p(x), \forall x \in E .
$$

\section{Prueba}

Antes de todo, veamos que de (1) y (2) se sigue que $p(x) \geq 0, \forall x \in E$.

En efecto, de (2) resulta que

$$
p(0)=p(0+0) \leq 2 p(0)
$$

consecuentemente $p(0) \geq 0$. De (1) resulta que $p(x)=$ $p(-x)$ y así mismo, $\forall x \in E$,

$$
2 p(x)=p(x)+p(-x) \geq p(x+(-x)) \geq p(0) \geq 0 \text {. }
$$

Primero en el caso real, es decir $\mathbb{K}=\mathbb{R}$. En este caso la hipótesis nos garantiza que

$$
\varphi(x) \leq p(x) \forall x \in G .
$$

El teorema anterior garantiza la existencia de una funcional lineal $\tilde{\varphi}: E \mapsto \mathbb{R}$ que extiende $\varphi$ a $E$ y que satisface

Ahora en el caso complejo $\mathbb{K}=\mathbb{C}$. En este caso $E$ es un espacio vectorial complejo y $\varphi$ toma valores en $\mathbb{C}$.

Definiendo $\varphi_{1}, \varphi_{2}: E \mapsto \mathbb{R}$ por

$$
\begin{aligned}
& \varphi_{1}(x)=\operatorname{Re}(\varphi(x)), \\
& \varphi_{2}(x)=\operatorname{Im}(\varphi(x)),
\end{aligned}
$$

es claro que $\varphi_{1}$ y $\varphi_{2}$ son lineales, toman valores reales y $\varphi=\varphi_{1}+i \varphi_{2}$. Como artificio, llamaremos a $E_{\mathbb{R}}$ y $G_{\mathbb{R}}$ los espacios vectoriales reales subyacentes a $E$ y $G$ (es decir, como conjunto del espacio en si mismo, las operaciones de adición es la misma y la multiplicación por escalar es con escalares reales). Entonces $\varphi_{1}$ y $\varphi_{2}$ son funcionales lineales sobre $G_{\mathbb{R}}$. Para $x \in G_{\mathbb{R}}$ obtenemos

$$
\varphi_{1}(x) \leq\left|\varphi_{1}(x)\right| \leq|\varphi(x)| \leq p(x) .
$$


Por el teorema anterior existe entonces una funcional lineal $\tilde{\varphi}_{1}: E_{\mathbb{R}} \mapsto \mathbb{R}$ que extiende $\varphi_{1}$ a $E_{\mathbb{R}}$ y que satisface $\tilde{\varphi}_{1} \leq p(x), \forall x \in E_{\mathbb{R}}$. Evaluaremos ahora el caso de $\varphi_{2}$. Note que para $x \in G$,

$$
\begin{aligned}
i\left(\varphi_{1}(x)+i \varphi_{2}(x)\right) & =i \varphi(x)=\varphi(i x) \\
= & \varphi_{1}(i x)+i \varphi_{2}(i x) \\
= & i \varphi_{1}(x)-\varphi_{2}(x) .
\end{aligned}
$$

Por tanto $\varphi_{2}(x)=-\varphi_{1}(i x) \forall x \in G$. Definiendo entonces

$$
\begin{gathered}
\tilde{\varphi}: E \mapsto \mathbb{C} \\
x \mapsto \tilde{\varphi}(x)=\tilde{\varphi}_{1}(i x)-i \tilde{\varphi}_{1}(i x),
\end{gathered}
$$

se tiene que $\tilde{\varphi}(x)=\varphi(x) \forall x \in G$.

Probemos que $\tilde{\varphi}$ es una funcional lineal en el espacio complejo $E$. En efecto

$\tilde{\varphi}(x+(a+b i) y)=\tilde{\varphi}(x)+(a+b i) \tilde{\varphi}(y) \forall x, y \in E$ y dado

$(a+b i) \in \mathbb{C}$.

$$
\tilde{\varphi}(x) \leq p(x), \forall x \in E
$$

De la desigualdad de (2) tenemos

$$
\begin{aligned}
-\tilde{\varphi}(x)=\tilde{\varphi}(-x) & \leq p(-x)=(-1) p(x)=p(x), \forall x \\
& \in E .
\end{aligned}
$$

Luego $|\tilde{\varphi}(x)| \leq p(x), \forall x \in E$.

$$
\begin{aligned}
& \tilde{\varphi}(x+(a+b i) y)=\tilde{\varphi}_{1}(x+(a+b i) y)-i \tilde{\varphi}_{1}(i(x+ \\
& (a+b i) y)) \\
& \tilde{\varphi}(x+(a+b i) y)=\tilde{\varphi}_{1}(x+(a+b i) y)-i \tilde{\varphi}_{1}(i x+ \\
& i(a+b i) y) \\
& \tilde{\varphi}(x+(a+b i) y)=\tilde{\varphi}_{1}(x)+\tilde{\varphi}_{1}((a+b i) y)- \\
& i \tilde{\varphi}_{1}(i x)-i \tilde{\varphi}_{1}(i(a+b i) y) \\
& \tilde{\varphi}(x+(a+b i) y)=\tilde{\varphi}_{1}(x)-i \tilde{\varphi}_{1}(i x)+\tilde{\varphi}_{1}((a+ \\
& b i) y)-i \tilde{\varphi}_{1}(i(a+b i) y) \\
& \tilde{\varphi}(x+(a+b i) y)=\tilde{\varphi}(x)+(a+b i)\left[\tilde{\varphi}_{1}(y)-\right. \\
& \left.i \tilde{\varphi}_{1}(i y)\right] \\
& \tilde{\varphi}(x+(a+b i) y)=\tilde{\varphi}(x)+(a+b i) \tilde{\varphi}(y) .
\end{aligned}
$$

Mostraremos finalmente que $|\tilde{\varphi}(x)| \leq p(x) \forall x \in E$.

Si $\tilde{\varphi}(x)=0$, entonces la desigualdad es $p(x) \geq$ 0 .Tomemos $x \in E$ tal que $\tilde{\varphi}(x) \neq 0$. Entonces existe $\theta$ tal que $\tilde{\varphi}(x)=|\tilde{\varphi}(x)| e^{i \theta}$. Se sigue que

$$
|\tilde{\varphi}(x)|=\tilde{\varphi}(x) e^{-i \theta}=\tilde{\varphi}\left(x e^{-i \theta}\right)
$$

Como $|\tilde{\varphi}(x)|$ es real por (1) tenemos

$$
\begin{gathered}
|\tilde{\varphi}(x)|=\tilde{\varphi}\left(x e^{-i \theta}\right)=\tilde{\varphi}_{1}\left(x e^{-i \theta}\right) \leq p\left(x e^{-i \theta}\right) \\
=\left|e^{-i \theta}\right| p(x)=p(x) .
\end{gathered}
$$

Extensión de las funcionales lineales en espacios normados

Proposición (Extensión de funcionales lineales en espacio normados) Sea $E$ un espacio normado. Para todo $x_{0} \in E, x_{0} \neq 0$, entonces existe una funcional lineal $\varphi \in E^{\prime}$ talque $\|\varphi\|=1$ у $\varphi\left(x_{0}\right)=\left\|x_{0}\right\|$.

\section{Prueba}

En efecto. Sea $x_{0} \in X, x_{0} \neq 0, Y \equiv x \mathbb{K}, \quad y=\alpha x$. Entonces $Y \subset X$ es un subespacio de $X$.

Definimos $\varphi: Y \mapsto \mathbb{K}$ talque

$$
y \mapsto \varphi(y)=\alpha\left\|x_{0}\right\|, y=\alpha x_{0} \forall \alpha \in \mathbb{K} .
$$

Entonces $\varphi$ es una funcional lineal acotada.

En efecto

a) $\varphi\left(c y_{1}+y_{2}\right)=\varphi\left(c \alpha x_{0}+\beta x_{0}\right)$

$$
\begin{aligned}
& \varphi\left(c y_{1}+y_{2}\right)=\varphi\left((c \alpha+\beta) x_{0}\right) \\
& \varphi\left(c y_{1}+y_{2}\right)=(c \alpha+\beta)\left\|x_{0}\right\| \\
& \varphi\left(c y_{1}+y_{2}\right)=c \alpha\left\|x_{0}\right\|+\beta\left\|x_{0}\right\| \\
& \varphi\left(c y_{1}+y_{2}\right)=c \varphi\left(y_{1}\right)+\varphi\left(y_{2}\right) .
\end{aligned}
$$

Por lo tanto $\varphi$ es lineal.

b) $\varphi$ es acotado. En efecto,

$$
|\varphi(y)|=\left|\alpha\left\|x_{0}\right\|\right|=\left\|\alpha x_{0}\right\|=\|y\| .
$$


Se sigue que

$$
\sup _{\substack{y \neq 0 \\ y \in Y}} \frac{|\varphi(y)|}{|y|}=\sup _{\|y\|<1}|\varphi(y)|=\sup _{\|y\|=1}|\varphi(y)|
$$

Entonces

$$
\|\varphi\|=\sup _{\substack{y \neq 0 \\ y \in Y}} \frac{|\varphi(y)|}{|y|}=\sup _{\substack{y \neq 0 \\ y \in Y}} \frac{\|y\|}{\|y\|}=1
$$

Por lo tanto $\|\varphi\|=1$.

Ahora por el teorema de extensión de funcionales $\exists \tilde{\varphi}: E \mapsto \mathbb{K}$ funcional talque $\left.\tilde{\varphi}\right|_{Y}=\varphi$ y $\|\tilde{\varphi}\|=\|\varphi\|=$ 1. Por otro lado $\tilde{\varphi}\left(x_{0}\right)=\varphi\left(x_{0}\right)=\left\|x_{0}\right\|$, en consecuencia $\forall x \neq 0, \exists \varphi \in X^{*}$ talque

$$
\|\varphi\|=1, \varphi\left(x_{0}\right)=\left\|x_{0}\right\|
$$

\section{CONCLUSIONES}

El Teorema de extensión de funcionales lineales permite asegurar la existencia sobre un espacio vectorial o un espacio normado, de funcionales lineales que satisfagan adecuadas propiedades, construyendo un subespacio adecuado, definiendo una funcional, sobre ese subespacio verificando las condiciones requeridas.

El teorema de extensión de funcionales lineales como generador de funcionales hace que dicho teorema se encuentre en la base de la teoría de la dualidad de espacios normado.

\section{REFERENCIAS BIBLIOGRÁFICAS}

BAGGETT, L. (1992). Functional analysis. New York: Marcel Dekker.

BRÉZIS, H. (1964). Análisis funcional Teoría y aplicaciones. Madrid: Alianza.

CABELLO, J. (2009). Análisis Funcional. España: Universidad de Granada.

CAROTHERS, N. (2000). Real Analysis. New York: Cambridge University Press.

GATICA, G. (2012). Introducción al Análisis funcional: Teoría y Aplicaciones. Parte 1. Chile: CIMA Universidad de Concepción.

KREYZIG, E. (1989). Introductory Functional Analysis with Applications. New York: Wiley Classics.

LAGES, E. (2014). Álgebra Linear. Río de Janeiro: IMPA.

LAROTONDA, G. (2017). Análisis Funcional. Un curso avanzado. Buenos Aires: Universidad de Buenos Aires.

LLORENS, E. (2016). Análisis funcional. España: Universitat de Valencia.

SEYMOUR, P. D. (1970). Teoría y problemas de Topología general. México: McGraw-Hill.

WAWRZYNCZYK, A. (1993). Introducción al Análisis Funcional. México: UAM-Unidad Iztapalpa. 